has a profound influence on the infection. There is no doubt also that the simple administration of this drug does not save all the patients, and it is necessary, therefore, to consider whether we can supplement its beneficial action. It has been shown that a specific antiserum acts synergically with sulphanilamide in streptococcal infections, and that in vitro pneumococcal serum acts in the same way with $M$ \& B 693. No reports have yet been made regarding the united action of pneumococcal serum and $M$ \& B 693, but there is little doubt that this will be a good combination. However, pneumococcal serum is not available in all types, it is expensive, and it is not always easy to administer in general practice.

It would seem that there is more hope in vaccine therapy. Vaccines are available for every type of pneumococci, or a polyvalent vaccine can be used which contains all of them. Vaccines are cheap and are easy to administer. It has been shown by many workers that even large doses of pneumococcus vaccine can be given in cases of pneumonia without risk of a negative phase, and Wynn has for many years been using stock vaccines as a routine treatment of pneumonia. Barach (1931) has shown that three days after the administration of pneumococcal vaccine to patients suffering from pneumonia specific antibodies can be demonstrated in their serum. Maclean, Rogers, and Fleming (1939) have demonstrated that mice which had received a single dose of pneumococcal vaccine and did not show a high degree of immunity recovered when treated with $M$ \& B 693, whereas the unvaccinated mice infected with the same dose of pneumococci and receiving the same treatment with M \& B 693 all died.

These observations, taken together, make a good case for the use of pneumococcal vaccines in pneumonia in man in conjunction with $M$ \& $B$ 693. If possible the pneumococcus should be typed and a vaccine of the specific type administered, but if typing is not possible then a stock polyvalent vaccine can be administered at once; and of course the sooner the vaccine is given the better the result is likely to be.

\section{Failures and Successes in Vaccine Therapy}

One sometimes hears that in a particular case or condition vaccine therapy has been tried and has failed. It is well to remember that there is vaccine therapy and vaccine therapy, and this may be illustrated by the following cases.

The first case was that of an American doctor practising as a specialist in diseases of the chest in one of the Western States. He had worked in our laboratories at St. Mary's for long periods before and during the war, and when he returned home he contracted severe influenza during the 1918-19 epidemic. After recovery he suffered from typical spasmodic asthma. He tried the ordinary remedies and climatic treatment; then he had his tonsils removed in New York, and there he was treated with a vaccine made from bacteria isolated from his sputum-all without benefit. Here, then, was a case in which vaccine therapy had been tried and failed. Fortunately he thought he would come to London to see whether his old colleagues at St. Mary's could help him. Immediately on arrival at Waterloo Station he had a severe asthmatic attack, so it could not be said that the London climate suited him. Examination of his sputum showed organisms, but nothing that seemed likely to account for his condition. The faeces were then examined, and an almost pure culture of a haemolytic enterococcus was found. He was treated with a vaccine of this organism in doses beginning with one-third of a million and increasing eventually to 1,000 millions. Apart from an attack soon after treatment was started he had no more asthma, and he is still free some seventeen years afterwards.
When he was convinced that he was recovering he sent the news to a fellow practitioner in the Western States who suffered from asthma with a similar history. She travelled some 6,000 miles to have a vaccine made from the haemolytic enterococci in her stool, for she also had a great preponderance of this organism. On the boat on the way over she found another asthmatic with a similar history. He, too, had an intestine full of enterococci, and a vaccine was successful in his case also.

These three cases happening together, all due to an enterococcal infection of the gut-a condition that is usually neglected-could not but make a lasting impression ; but the moral of the tale is that in a particular case vaccine therapy was tried and had failed, and yet when it was tried again in another way it was eminently successful. This case could, I am sure, be paralleled by hundreds of others.

In conclusion I must thank my colleagues, and especially Drs. Maclean and Young, for permission to make use of work in which they took a considerable part.

\section{REFERENCES}

Barach, A. L. (1931). J. exp. Med., 53, 567

Cokkinis, A. J., and McElligott, G. L. M. (1938), Lancet, 2, 355. Fleming, A. (1938). Ibid., 2, 74

Grinnell, F. B. (1932). J. exp. Med., 56, 907

Loewenthal, H. (1939). Lancet, 1,197 .

Maclean, I. H., Rogers, K. B., and Fleming, A. (1939). Ibid., 562. Shope, R. E. (1937). J. exp. Med., 66, 169.

\section{DERMATITIS FROM SULPHONAMIDE COMPOUNDS}

\section{BY}

DAVID ERSKINE, M.D., D.P.H.

Dermatologist to the Dreadnought Hospital; Medieal Officer in the Venereal Diseases and Dermatological Departments, Guy's Hospital

The extensive use of the sulphonamide group of drugs has resulted in a certain incidence of toxicity. The various manifestations of this toxicity have been reported in the literature from time to time, but comparatively little attention has so far been drawn to the skin eruptions that may be encountered during the administration of the drugs. These eruptions often raise problems of differential diagnosis, and their variations in type and degree and in their response to treatment are not without interest. A brief review is here attempted of some thirty-six cases of sulphonamide dermatitis seen chiefly at Guy's Hospital during a fairly extensive administration of these drugs in the venereal diseases and dermatological departments over the last three years. The cases are surveyed from a clinical aspect, and certain information which is now considered necessary has frequently not been recorded, as it was only during prolonged observation that various characteristics of the eruptions became more clearly appreciated. Nevertheless, it has been felt that the information available may be of some value in correlating the toxicity of the drugs.

Newman and Sharlit (1937) reported four cases of light sensitization following sulphonamide administration; the eruptions occurred on the exposed areas, between the second and fifth days; they were macular in type, with raised papules and oedema. The eruptions faded during five to six days of protection from light, and could be reproduced on giving further sulphonamide without exposure to light. These cases also exhibited patch sensitivity. Menville and Archinard (1937) reported similar cases occurring after large doses of prontosil-one case escaping eruption after exposure to sunlight on the ninth 
day of administration but succumbing after exposure on the tenth day. Hallam (1939) has reported a case which illustrates the danger of ultra-violet light to patients taking sulphonamide. Rimington and Hemmings (1938) have shown that an increased output of porphyrin occurs during sulphanilamide therapy together with coproporphyrin I, which is a photosensitizer; they consider that in every patient this process varies in degree according to individual factors, and this variation may account for the relative infrequency of the light-sensitization type of eruption. Wien (1938) found that in animals 2-sulphanilylaminopyridine (M \& B 693) does not show the same tendency to increased porphyrinuria.

Salvin (1937) reported a generalized urticarial manifestation with no allergic family history in a patient who gave a positive patch reaction. Schonberg (1937) records an urticarial eruption which progressed after discontinuance of the drug and then developed into a generalized purpuric rash. A single tablet one month later produced an irritating scarlatiniform eruption. Frank (1937) reports a similar relapse from one dose given six days after improvement from a generalized morbilliform sulphonamide eruption. Goodman and Levy (1937) describe purpuric spots with generalized maculo-papular eruptions in patients who relapsed immediately on later administration, but who gave negative patch reactions. Finney (1937) mentions a case of morbilliform eruption with albuminuria which slowly cleared on stopping the drug. Exfoliative dermatitis after 86 grammes of sulphonamide with severe general toxic symptoms was reported by Myers and others (1937). Davis (1939) reports a morbilliform eruption on the fourteenth day which did not clear on ceasing to administer the drug. This case developed later a superadded scarlatiniform rash, but since there was also severe stomatitis the later eruption may possibly have been a toxic manifestation of the stomatitis, especially as he reports that the rashes cleared immediately the stomatitis improved. This case may therefore further illustrate the tendency of one eruption to predispose to the occurrence of another. Palmer and Hanbury (1939) report an eruption bearing a similarity to the irregular flat-topped papules of lichen planus.

\section{Details of Cases}

The majority of the cases have shown a generalized eruption of the morbilliform type, but rashes of urticarial, scarlatiniform, and light-sensitization type have been observed on several occasions. In almost every case the skin manifestation has appeared between the eighth and fourteenth days and most frequently between the eighth and tenth days. The occurrence of the rash apparently depends more on this time factor than on the dose of the drug administered. The possibility of a sulphonamide preparation producing an eruption seems to be present, whatever compound is used, if the administration is continued for more than eight days, as is so often necessary ; but its frequency would appear to depend to some extent on the therapeutic efficiency of the particular preparation used. In this series, however, the light-sensitization type of eruption has only been observed primarily with sulphanilamide.

Although generally it has been necessary to discontinue the administration of the sulphonamide when the rash has appeared, it has been possible in some cases to continue the drug and to observe a rapid clearing of the eruption. With certain cases a further course of sulphonamide has been instituted at a later date with the same or different compound of the group. Here again the variations in response have been surprising, for some cases show an immediate sensitivity to the substance, others develop a rash after another latent period, while a further group complete this later course without skin manifestations.

Details of the individual cases are given in the table. The morbilliform type of rash has usually begun as generalized irritation, followed in a few hours by faint bluish macules on the upper arms or trunk, increasing in intensity and spreading to cover the front and back of the trunk, while the limbs show a profuse eruption on the extensor and on the more proximal regions. These macules often become slightly raised, and the rash may progress to become a maculo-papular eruption, in some cases with fine scaling, and frequently exhibiting a degree of urticarial response. The glands are not usually involved, and the mucous membranes usually escape, although there may be coryza and lacrimation, while vesiculation has been reported. Frequently there is considerable malaise, with a pyrexia of $3^{\circ}$ or $4^{\circ}$ and a slight increase in the pulse rate. Scarlatiniform and erythematous eruptions are generally extensive, irritation is the rule, but circumoral pallor is not seen; in the absence of a primary throat infection there is no congestion of the fauces.

The simple urticarial eruptions have shown patches of erythema with severe irritation, the appearance of wheals, and areas of considerable oedema. The eruption in the three cases showing light sensitization was limited to the face, neck, forearms, and hands, extending just beyond the exposed areas, and there was considerable oedema, with macules coalescing to form intense erythema with vesiculation.

\section{Differential Diagnosis}

Several of these patients have withheld from their private doctors information that they were taking tablets, with the result that they have occasionally, and not surprisingly, been diagnosed as cases of German measles, measles, scarlet fever, enema rash, etc., and have sometimes been sent to fever hospitals as such. Cleveland (1939) reports a case where variola was for a time suspected.

A correct diagnosis depends primarily on the recognition that sulphonamide compounds may produce skin manifestations of varying forms and that the compounds are now administered for conditions which may lead the patient to conceal that he has been taking them. The exclusion of the exanthemata may then give some difficulty, although the frequency of irritation and the infrequency of any of the signs which commonly accompany the specific fevers may be helpful. A skin eruption between the eighth and fourteenth days of known sulphonamide administration is highly suggestive of a sulphonamide dermatosis. Intradermal sensitization tests are not reliable: they probably depend on the cause of the particular eruption, and even in truly sensitized cases the skin may already be reacting to its maximum capacity. Urine testing may be of more help, but the amount of sulphonamide excreted varies considerably: some of the eruptions appear to be associated with a retention of the drug, while in others elimination in the urine is normal. Werner (1939) described a simple test, using 3 per cent. $p$-dimethylamino-benzaldehyde in 10 per cent. hydrochloric acid. One part of this solution added to nine parts of diluted urine gives a yellow flavine-like colour or a turbid yellow precipitation, according to the concentration of the drug, in the presence of sulphonamide. Lloyd and Lloyd (1930) drew attention to the diazo test first reported by Abelin (1911), but using resorcinol as the second component. This test, 
Analysis of 36 Cases of Dermatitis from Sulphonamide Compounds

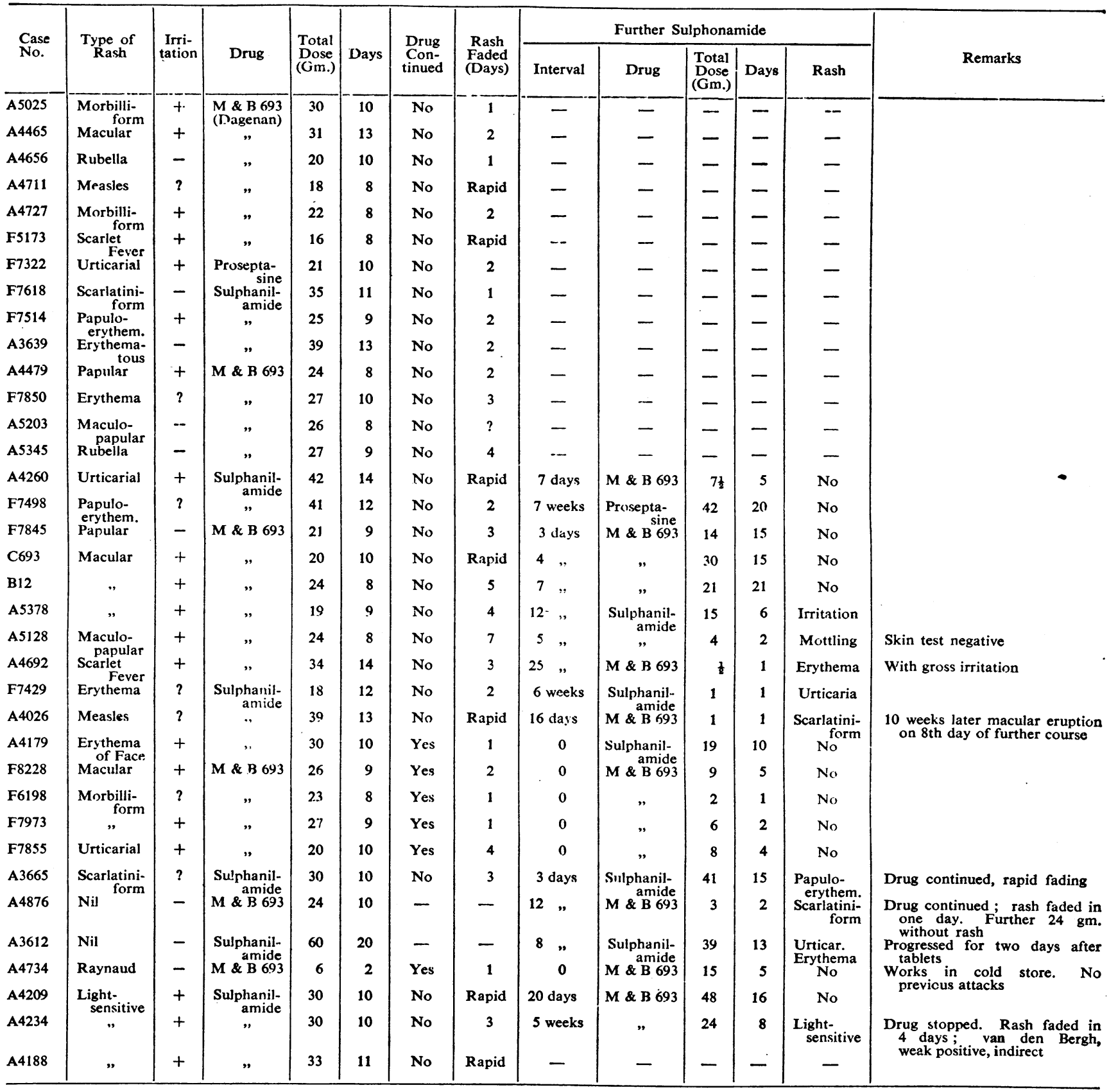

after diazotization of the sulphonamide compound in the urine, will give a port-wine colour with several of the more commonly used sulphonamide preparations. It is nevertheless important to remember that a sulphonamide rash may be due to retention of the drug in the body, so that the urine may fail to give a positive reaction (see below).

\section{Discussion}

The varying skin manifestations and the differences of their later history during sulphonamide administration do not seem to agree with any single theory of causation, and this view has already been advanced by Tedder (1939). The appearance of a rash only after eight to fourteen days of treatment bears a similarity to the erythema of the ninth day noted so frequently with the arsenobenzene compounds. It is also reminiscent of such an allergic phenomenon as the serum rash seen after approximately the same interval from serum administration, with the one difference that I have not heard of any case occurring after only one or two doses of a sulphonamide. In fact, to produce a rash the sulphonamide must be given up to the time of its appearance or at any rate the day preceding; even then this evidence of intolerance to the compound is observed in only approximately 10 per cent. of cases. Once a patient has shown a rash he may remain sensitive to the drug for months, and one tablet ( 0.5 gramme) taken after a long interval may produce a further eruption in a few hours, even when there is no previous history of any allergic manifestations in the patient or his family. Again, other cases take a further prolonged course of the drug without any incident. 
The fact that it is sometimes possible to demonstrate a normal elimination of the substance in the urine while the rash is present suggests that there may be cases of true sensitization to the drug, while in those showing a retention of the drug the rash may be a toxic result of failure to eliminate the compound that is continually being ingested. In this respect it is interesting to observe that examples of drug fever, appearing after a similar time interval but without any skin rash, have been not infrequently observed. When the elimination is at fault it has been found necessary to discontinue the drug in order to clear the skin. Some patients with evidence of sulphonamide in the urine have continued medication under observation, with the result that the skin has cleared more rapidly than in the other group, and in none of these cases has further eruptions been observed if the drug has been again administered at a later date. The patient may in this way have been desensitized to the drug. In reporting earlier cases (Erskine, 1938) I put forward a further theory for which there appears to be some support on the grounds of clinical response to therapy. It is conceivable that the drug, acting on some buried focus of infection in the body, liberates toxins from that focus, and that these toxins are the cause of the eruptions sometimes seen. Certainly some cases bear a striking resemblance to scarlet fever, even including pre-eruptive shivering, malaise, and nausea, and the skin manifestations of scarlet fever are at any rate believed to be due to a toxin. The increased eruption occasionally seen in psoriasis during sulphonamide treatment and the response of certain cases of lupus erythematosus (Barber, 1938) might be considered to support this view.

Epstein (1939) has drawn attention to the need for distinguishing true light sensitization from an eruption of that nature seen during sulphonamide therapy for which he has suggested the term of "photo-allergy"; but Rimington and Hemmings have shown that sulphanilamide does produce an increased porphyrin excretion, evidence which suggests true photo-sensitization in these cases. It is well known that during sulphonamide administration an excess of urobilin can often be demonstrated in the urine, and one of my cases gave an indirect positive van den Bergh reaction.

In conclusion it is justifiable to remark that although these eruptions are encountered not uncommonly during treatment of a case with sulphonamide compounds they are not serious contraindications to chemotherapy. With careful observation the eruptions are easily controlled, and diagnosis need not be difficult when the possibility of this evidence of sulphonamide intolerance is recognized. Artificial sunlight and unnecessary exposure to sunshine should, however, be forbidden a patient during the period of treatment, while he might be saved unnecessary embarrassment, especially if ambulatory, if the urine were tested frequently by these simple methods. Fewer patients might then be permitted to experience this (to them) startling complication. Finally, although certain compounds appear in the table to have an increased liability to the production of the rash, it is solely because we have found them, for our purposes, more effective therapeutically.

\section{Treatment}

The majority of eruptions will rapidly clear with appropriate treatment or cessation of administration. When the elimination of sulphonamide in the urine is found to be depressed the drug should be discontinued. Large quantities of fluid and alkaline diuretics will help rapid elimination. When there is no evidence of diminished excretion the drug may be continued under careful observation in the same or diminished dosage so long as no serious exacerbation of the rash occurs. This usually leads to a more rapid clearing of the skin in these cases, and appears possibly to desensitize the patient.

Ephedrine may help to control severe irritation, especially when a pronounced urticarial element is present in any type of rash. Local application is of secondary importance in any but the most intense eruption, but a calamine lotion has been found the most helpful.

\section{Summary}

A brief review of the literature of dermatitis from sulphonamide compounds is given and theories of its causation are advanced.

Morbilliform, scarlatiniform, urticarial, and light-sensitization types of eruption occurring during the administration of sulphonamide compounds are reported.

The cases have been divided into two groups depending on urinary elimination, and appropriate lines of treatment are suggested.

I wish to thank Dr. H. W. Barber and Mr. V. E. Lloyd for their helpful criticism and for permission to report details of cases in their care.

\section{REFERENCES}

Abelin, J. (1911). Münch. med. Wschr., 58, 1002, 1771.

Barber, H. W. (1938). Lancet, 2, 668.

Cleveland, D. E. H. (1939). Arch. Derm. Syph., Chicago, 39, 693. Davis, E. (1939). Lancet, 1, 1042

Epstein, S. (1939). Arch. Derm. Syph., Chicago, 39, 225.

Erskine, D. (1938). Brit. J. vener. Dis., 14, 39.

Finney, J. O. (1937). J. Amer. med. Ass., 109, 1982.

Frank, L. J. (1937). Ibid., 1011.

Goodman, M. H., and Levy, C. S. (1937). Ibid., 1009.

Hallam, R. (1939). British Medical Journal, 1, 559.

Lloyd, N. L., and Lloyd, V. E. (1930). Brit. J. vener. Dis., 6, 1. Menville, J. G., and Archinard, J. J. (1937). J. Amer. med. Ass., 109, 1008 .

Myers, G. B., Heide, E. C. V., and Balcerski, M. (1937). Ibid., 1983.

Newrnan, B. A., and Sharlit, H. (1937). Ibid., 1036.

Palmer, G. W., and Hanbury, D. R. (1939). British Medical Journal, 1 ' 561 .

Rimington, C., and Hemmings, A. W. (1938). Lancet, 1, 770.

Salvin, M. (1937). J. Amer. med. Ass., 109, 1038.

Schonberg, I. L. (1937). Ibid., 1035.

Tedder, J. W. (1939). Arch. Derm. Syph., Chicago, 39, 217.

Werner, A. E. A. (1939). Lancet, 1, 18.

Wien, R. (1938). Quart. J. 'Pharm. and Pharmacol., 11, 217.

Copies have been received from the British Pharmacopoeia Commission of two reports by its Committee on Pharmaceutical Chemistry. These are chiefly concerned with corrections of assays and of tests of purity. In addition new monographs have been prepared for the assay of several important drugs, including halibut-liver oil, cyclopropane, mandelic acid, and sulphanilamide. The Commission is issuing these reports in order that the recommendations made therein may be, available for discussion before they are adopted for the next edition of the British Pharmacopoeia. Such discussion will largely be confined to analytical chemists, since most of the recommendations relate to details of technique. The reports provide evidence of the quantity and quality of the voluntary work carried out by the committee. The importance of this work is very great, because the reputation of the British Pharmacopoeia as a presumptive legal standard depends upon the accuracy of the chemical methods which it recommends. The reports (numbered respectively 11 and 12) are published from the office of the General Medical Council, 44, Hallam Street, London, W., price 1s. 6d. each. 